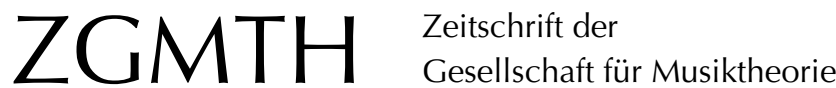

Spurný, Lubomír (2007): Was ist neu an Hábas Neuer Harmonielehre? ZGMTH 4/3, 323-328. https://doi.org/10.31751/264

C 2007 Lubomír Spurný

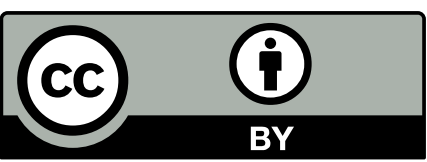

Dieser Text erscheint im Open Access und ist lizenziert unter einer Creative Commons Namensnennung 4.0 International Lizenz.

This is an open access article licensed under a

Creative Commons Attribution 4.0 International License.

veröffentlicht / first published: 01/07/2007

zuletzt geändert / last updated: 04/07/2009 


\title{
KOLUMNE
}

\section{Was ist neu an Hábas Neuer Harmonielehre?}

\author{
Lubomír Spurný
}

Die 20er Jahre des vergangenen Jahrhunderts waren eine bedeutende Zeit für die Entwicklung der Musiktheorie in Tschechien. Deutlich wird dies an zwei höchst unterschiedlichen, doch gleichermaßen wichtigen Bucherscheinungen jener Jahre: Die Harmonielehre von Otakar Šín (1922) gründete als erste Veröffentlichung ihrer Art auf der `Funktionstheorie` Hugo Riemanns; die Neue Harmonielehre ${ }^{1}$ von Alois Hába (1927) indessen nahm ihren Ausgang in der Untersuchung akustischer Grundlagen. Während Šíns Buch die künftige Entwicklung der Harmonielehre und die Lehrtätigkeit in Tschechien stark beeinflusste, erfuhr dasjenige Hábas, das im Ausland zumeist nur als Beispiel einer randständigen nationalen Tradition Erwähnung gefunden hat, keine nachhaltige Rezeption. Auch das kompositorische Werk Hábas (1893-1973) ist heutzutage so gut wie unbekannt. Es findet fast nur noch Erwähnung im Zusammenhang mit mikrotonaler und athematischer Musik.

Mit Franz Schrecker, seinem Lehrer aus Wiener Tagen, war Hába im Herbst 1920 nach Berlin gekommen und lebte dort mit Unterbrechungen bis zum Frühling 1923. Hábas, dessen Originalität bereits während seiner Wiener Zeit für Aufsehen gesorgt hatte, fand sich schnell in ein künstlerisches Milieu ein, das ihm vielfältige Anregungen bot: `Neur zu sein, war im Berlin jener Tage ein gefragtes Attribut. Hába griff die verschiedenen Entwicklungstendenzen der zeitgenössischen Musik auf, suchte aber zugleich nach dem eigenem Ausdruck und den eigenen technischen Lösungen. Am Ende stand - ganz dem Selbstverständnis des erst jungen `demokratischen Zeitalters` verpflichtet - sein Konzept einer >Musik der Freiheit`. Im Folgenden möchte ich zeigen, dass in ähnlicher Weise auch seine Musiktheorie durch eine höchst individuelle Auseinandersetzung mit zeitgenössischen Ansätzen und Denkweisen geprägt ist.

`Neues`, das die Bedeutung der bisherigen Lehre annulliert, kann in einem Lehrbuch wie einer Harmonielehre, das vom Prinzip her zumeist konservativ ist, nur allzu leicht exzessiv wirken. Darauf spielte Otakar Šín an, als er die Neue Harmonielehre als ein »äußerst persönliches Werk« bezeichnete. ${ }^{2}$ Šín hatte unbestritten Recht: Hábas Arbeit ist kein

1 Hábas Neue Harmonielehre des diatonischen, chromatischen, Viertel-, Drittel-, Sechstel- und Zwölfteltonsystems erschien 1927. Den bereits 1925 entstandenen, ursprünglich tschechischen Text übersetzte der Autor selbst, die Textrevision unternahm Erich Steinhardt.

2 Šín 1927, 125. 
Lehrbuch im klassischen Sinn. Ihrem Autor gibt es vor allem Gelegenheit, sein Konzept einer >Musik der Freiheitin Begriffe der Musiktheorie zu fassen. Dahinter steht ein schöpferisches Ideal, das den gänzlich freien Willen des Autors zum Programm erhebt. Bereits in Hábas Studie Grundlagen der Tondifferenzierung (1925) ist hierzu folgender Hinweis zu finden: „Das einzige allgemeine Kriterium für die Berechtigung, Wahrhaftigkeit und Aufrichtigkeit des musikalischen Schaffens überhaupt, beruht darin, dass der schöpferische Musiker lediglich die Musik schreibt, die er sich vorstellen kann.« ${ }^{3}$ Diese Überlegung bestimmt nicht nur den so genannten »nichtthematischen Stil« von Hábas Kompositionen jener Jahre, sondern liegt auch seiner Harmonielehre zugrunde. Hába gibt kein Konglomerat von Verboten und Regeln an. Vielmehr ließe sich etwas überspitzt sagen: Das einzig wirkliche Gesetz lautet, snicht traditionell zu sein`. Nahezu jedes der im Buch dargestellten technischen Verfahren scheint gegen die Tradition gerichtet zu sein. So nehmen viele der dargelegten Akkordbildungen ihren Ausgang von Hábas Behauptung, es existiere kein separates »konstruktives Gesetz für die Harmoniebildung«, vielmehr bilde »die Leiter [...] das gemeinsame Gesetz für die Melodie- und Harmoniebildung «. ${ }^{4}$ Jeder auf der jeweiligen Leiter fußende Klang sei legitim ${ }^{5}$ und für Akkordverbindungen gäbe es keine allgemeinen Gesetze. Die Vorschläge Hábas für die Verbindung von Klängen sind vor allem durch die Aufforderung gekennzeichnet, gemeinsame Töne zwischen den einzelnen Klängen zu vermeiden, wodurch ein permanenter Wechsel zwischen möglichst unterschiedlichen Klängen erreicht werden soll. Auch wenn von `Tonalitätı explizit keine Rede (mehr) ist, herrscht hier dennoch eine spezifische Systematik, durch welche die klanglichen Verhältnisse reguliert werden, die so genannte sTonzentralität: Melodische Linien und akkordische Progressionen orientieren sich an einem festen `Tonzentrum‘, das durch chromatische Nachbartöne profiliert und gestärkt wird. Ausgesprochen antitraditionell sind schließlich auch die Ausführungen zur mikrotonalen Harmonik. Die Tatsache, dass es sich im Falle des Viertel-, Drittel-, Sechstel- und Zwölfteltonsystems um eine Applikation von Regeln der ursprünglichen Diatonik und Chromatik handelt, ändert nichts an der Neuartigkeit der hier diskutierten Verfahren.

Dennoch: Hábas Neue Harmonielehre ist kein Buch ohne Vorgeschichte, ja, es gewinnt sogar an Bedeutung, betrachtet man es im Kontext des musiktheoretischen Denkens der Zeit. Das gilt zunächst für seine Beziehung zur tschechischen Musiktheorie, vor allem zu jenem Zweig, der nicht (oder noch nicht) unter dem Einfluss von Hugo Riemann stand (Otakar Hostinský, František Zdeněk Skuherský, Karel Stecker, Leoš Janáček). ${ }^{6}$ Auch bestehen inhaltliche Bezüge zu Die Lehre von der Harmonik der diatonischen, der ganztonigen und der chromatischen Tonreihe von Hábas Brünner Landsmann Bruno Weigl (1925). ${ }^{7}$ Nicht zuletzt aber lassen sich Verknüpfungen zur Gedankenwelt Arnold

3 Hába 1925, 55.

4 Hába 1927, 24 f.

5 Es werden nur Zusammenklänge mit gleichbleibenden Intervalldistanzen systematisch dargestellt.

6 Vgl. Hostinský 1879, Skuherský 1885 sowie Janáček 1897 und 1912.

7 Darüber hinaus finden sich auch außereuropäische Einflüsse: In der Zeit von Hábas Berliner Aufenthalt ermöglichte ihm der stellvertretende Direktor der Berliner Hochschule, Georg Schünemann, den Besuch des Phonogramm-Archivs, wo der Komponist für seine athematische und mikrotonale 
Schönbergs herstellen. Schönberg studierte nachweislich Hábas Neue Harmonielehre ${ }^{8}$ und empfahl die Anschaffung einer deutschen Ausgabe für die Bibliothek der Harvard University. Anhand eines im Arnold Schönberg Center Wien verwahrten Exemplars lässt sich Schönbergs Interesse an Hábas Harmonielehre konkret fassen: Schönberg hat es eigenhändig mit überwiegend kritischen Anmerkungen versehen. ${ }^{9}$ Sie betreffen fast ausschließlich Partien im ersten Teil des Buches, die sich explizit auf seine eigene Person beziehen, und zielen darauf, Hábas Schönberg-Bild zu korrigieren. Den Teil über Mikrointervalle lässt er unkommentiert. ${ }^{10}$

Hábas Harmonielehre zeugt von einer sehr eigenständigen Schönberg-Rezeption. Hinweise auf Schönberg sind im Buch sehr häufig zu finden; vor allem Themen und Fragestellungen, die Schönberg bereits in seiner Harmonielehre angesprochen hatte, werden von Hába, wenn auch zumeist mit anderen Schlussfolgerungen, aufgegriffen. Vielfach werden Gedanken Schönbergs einer bisweilen polemischen Kritik unterzogen. Gleichzeitig versucht Hába eine eigenständige Auffassung zu entwickeln, indem er das musikalische Denken Schönbergs in seinem eigenen Sinne interpretiert. ${ }^{11}$ Hábas Bemühungen um eine Abgrenzung von Schönberg und der gleichzeitige Wunsch, eine gemeinsame Sprache mit ihm zu finden, werden bereits aus dem Vorwort ersichtlich. Dort beschäftigt sich der Autor zunächst allgemein mit der Problematik der ästhetischen Produktion. Im weiteren Verlauf geht es dann konkret um musikalische Fragestellungen: um den Dissonanzbegriff und die Bedeutung von Tonleitern oder Reihen als Grundlage der Komposition. ${ }^{12}$ Wenn Hába von unterschiedlichen Tonleitern spricht (Fünf-, Sechs- oder Elftonreihen), spürt man deutlich seine Tonalitätsauffassung, die die Möglichkeit von

Musik Beispiele aus dem Bereich der außereuropäischen Musik finden konnte. Vielleicht begannen hier Gedanken zu reifen, die später in Hábas Texten zum Ausdruck kommen: So meinte Hábas, die Musiken verschiedener Kulturen repräsentierten verschiedene Stufen einer Entwicklung; zwischen ihnen bestünden natürliche Bindungen. Ausgehend von einem Vergleich des Klangmaterials verschiedener Musikkulturen gelangte er außerdem zu der Überzeugung, auch die apriorischen Kategorien der europäischen Musik besäßen ebenso wenig ewige Geltung, wie die tradierten kompositorischen Mittel und Verfahrensweisen.

8 Dies geht aus einem Brief Schönbergs an Hugo Leichtentritt vom 3. Dezember 1938 hervor. Schönberg schreibt:»In der Universität hatte ich nicht Zeit, Ihre Frage nach deutschen Büchern, die mich interessieren, ausführlich zu beantworten. Ich bin kein `Leser und kenne daher eigentlich die folgenden Bücher nur sehr oberflächlich, und meistens nur einzelne aus dem Zusammenhang gerissene Abschnitte. Nichtsdestoweniger erinnere ich mich an manchen guten Gedanken. Voraussichtlich werden Sie ja selbst, wenn nicht alle, so die meistens davon kennen. Vor allem (obwohl ich mit fast allem nicht einverstanden bin): [...] « $(1958,222)$. Es folgt eine Auflistung von insgesamt 11 einschlägigen Titeln, darunter Hábas Harmonielehre. Freilich sollte die Bedeutung der Mitteilung Schönbergs nicht überschätzt werden: Er bewertet keines der zitierten Werke und räumt eine nur oberflächliche Lektüre ein, die für ein eher mechanisches Vorgehen bei der vorgenommenen Auswahl zu sprechen scheint.

9 Es gibt um die 20 Anmerkungen auf 13 Seiten des Buches. Der Sinn mancher seiner Äußerungen bleibt unklar, da der Buchrand unsachgemäß beschnitten wurde.

10 Von Mikrointervallen hatte Schönberg schon in seiner Harmonielehre geschrieben (1922, 23-25).

11 Hába war einer der Ersten (1925 bzw. 1927), die Schönbergs ’Prinzip der Komposition mit zwölf Tönen s positiv rezipierten. Auch später hob er den wertvollen Einfluss des Schönbergschen Schaffens stets hervor.

12 Besonders dieser Problematik widmet Schönberg etliche Anmerkungen. 
sAtonalität a ausschließt: Jedes Stück ist tonal, weil sein Tonmaterial notwendig ein Bestandteil einer `Reihe` ist. Von diesem Grundgedanken ausgehend entwickelt Hába eine Menge von Tonleitern, die sich durch die Anzahl der Töne und die Intervallfolge voneinander unterscheiden. Fragt man nach der Inspirationsquelle für Hábas Vorgehen, findet man die Antwort nicht bei Schönberg: Hába beruft sich vielmehr auf die modalen Besonderheiten der Volksmusik, die auch von anderen tschechischen Autoren erkannt und genutzt wurden (Novák, Janáček). Weitere Anregungen verdanken sich dem Werk Ferruccio Busonis. Zwar wird Busoni in der Neuen Harmonielehre nur am Rande erwähnt: »Für Busonis Ideen, die den Aufbau neuer Leitern betreffen (s. `Entwurf der neuen MusikÄsthetikı) habe ich mich lebhaft interessiert. «13 Doch war Hábas Kenntnis von Busonis theoretischem Werk keinesfalls oberflächlich: Die Gliederung der Neuen Harmonielehre und die zentralen Themenbereiche decken sich auffällig mit den »fünf Wegen«, welche die neue Musik, wie Busoni in seiner Abhandlung Die neue Harmonik(1911) ausführt ${ }^{14}$, einschlagen soll. Mit Bezug auf Debussy werden dort auch neuartige Tonleitern erwähnt: »Das erste neue harmonische Gebäude stützt sich auf die Akkordbildung nach gebräuchlichen Tonleitern (Debussy verwendet, von 113 Skalen, die ich zusammenstellte, nur die Ganztonleiter, und auch diese nur in der Melodie). « $^{15}$ Busonis Vision einer zukünftigen Harmonik, die sich mit dem Gebrauch neuer Skalen verbindet und darin Hábas Theorie sehr ähnelt, teilte Arnold Schönberg jedoch gerade nicht. ${ }^{16}$ Trotzdem bezieht sich Hába nicht auf Busoni, sondern auf Schönberg und dessen Zwölftönigkeit. Der Autor lässt keine einzige Gelegenheit aus, um die Ähnlichkeit eigener neuer Reihen mit der Schönbergschen `Grundgestalt‘ zu erwähnen: „Die Grundgestalt (im Schönbergschen Sinne) ist schon ein Kunstgebilde, der Leiterkonstruktion entnommen. ${ }^{17}$ An anderer Stelle heißt es: »Aus den leitereigenen Tönen jeder der 581 Leitern kann man viele Grundgestalten (im Schönbergschen Sinne) aufstellen und unzählige Melodien gewinnen. « ${ }^{18}$ Bemerkenswert ist, dass Schönberg in seinem Handexemplar diese recht willkürliche Vereinnahmung seiner eigenen Theorie unbeachtet lässt. Alle weiteren Erwähnungen seiner Person jedoch kommentiert er kritisch. So schreibt Hába etwa, es wäre »richtiger die Leiter als Grundgestalt zu bezeichnen. Die Grundgestalt, wie sie z. B. Schönberg meint, ist schon eine Ableitung von der Leiter-Grundgestalt, $[\ldots] . «^{19}$ Hier merkt Schönberg nur ein lapidar zweifelndes »Wo?« an. An anderer Stelle erwähnt Hába Schönberg im Zusammenhang mit der sakzidentellen Leiter, die in diesem Falle eine Terzenleiter ist, »welche nebenbei

13 Hába 1927, XIV.

14 Busoni 1922.

15 Ebd., 159.

16 In seiner Harmonielehre schreibt Schönberg: „Und Busoni, dieser vornehme und mutige Künstler; ich schätze und verehre ihn sehr. Aber die Plage, Hunderte von Skalen auszurechnen, könnte er sich ersparen. Ich habe mir mit Müh' und Not die Namen der sieben Kirchentonarten gemerkt; und das waren serst die Namen`! Ich werde mir nicht fünf von seinen Tonarten merken können. Wie soll ich sie aber dann komponieren - wenn ich sie gar nicht vor mir habe? Nein, so wie Weingartner möchte ich gar nichts machen!« $(1922,474)$

17 Hába 1927, 25.

18 Ebd., 124.

19 Ebd. 
bemerkt auch in engster Beziehung zu dem Prinzip der Schönbergschen Grundgestalten steht.« Hier fügt Schönberg ein »wieso?» hinzu. Hába fährt fort: »Also gelangen wir durch diese Erwägung wieder auch von dem Terzenbau zu der altgriechischen Theorie und zu der theoretischen Denkart Schönbergs. Der Unterschied zwischen einer C-Dur-Leiter, einer Terzenleiter, welche aus den sieben Tönen der C-Dur-Leiter besteht, und einer Schönbergschen Grundgestalt, welche gleichfalls aus den sieben Tönen der C-Dur-Leiter besteht, liegt nur in der Art der verschiedenen Reihenfolge der sieben Töne [...]. «"

Schönbergs Kommentare sind trotz ihrer Kürze vielsagend. Die von Hábas behauptete Ähnlichkeit der eigenen Reihen mit der Schönbergschen Grundgestalt oder gar eine Verwandtschaft von Schönbergs stheoretischer Denkartı mit der altgriechischen Theorie sind aus Schönbergs Sicht, und - so wäre zu ergänzen - nicht minder aus der unsrigen heutzutage, unzutreffend. Einen Hinweis darauf, warum Hába dennoch die Richtigkeit der eigenen Verfahrensweisen durch einen zweifelhaften Rekurs auf Schönberg zu erweisen versuchte, könnte das oben skizzierte Milieu geben, in dem sich Hába zur Zeit der Abfassung seines Buches bewegte. Die dort kursierenden Wertvorstellungen besaßen für die mitteleuropäische Avantgarde allgemeine Gültigkeit. Hinsichtlich der Verbindung ästhetischer Ideen und deren technischer ,Übersetzung، verkörperte Schönberg eine unbestrittene Autorität. Hába, wie viele Komponisten und musiktheoretischen Denker jener Zeit, fühlte sich von der Schönbergschen Gedankenwelt gleichzeitig bedroht und inspiriert. Nur so ist zu erklären, warum sich Hábas, um sein Plädoyer für eine `Musik der Freiheit‘ zu stützen, ausgerechnet auf Schönberg beruft, dessen ästhetische und kompositionstechnische Position von der seinen vergleichsweise weit entfernt war.

\section{Literatur}

Busoni, Ferruccio (1922), »Die neue Harmonik« [1911], in: Von der Einheit der Musik. Gesammelte Aufsätze, Berlin: Max Hesses Handbücher, 159-160.

Hába, Alois (1925), „Grundlagen der Tondifferenzierung und der neuen Stilmöglichkeiten in der Musik«, in: Von neuer Musik. Beiträge zur Erkenntnis der neuzeitlichen Tonkunst, Köln: Marcan, 52-58.

(1927), Neue Harmonielehre des diatonischen, chromatischen Viertel-, Drittel-, Sechstel- und Zwölfteltonsystems, Leipzig: Kistner \& Siegel.

Hostinský, Otakar (1879), Die Lehre von den Musikalischen Klängen, Prag: H. Dominicus.

Janáček, Leoš (1897), O stavbě souzvukův a jejich spojův [Über die Zusammensetzung der Akkorde und ihrer Verbindungen], Brno.

— (1912), Úplna nauka o harmonii [Vollständige Harmonielehre], Brno: A. Píša.

Schönberg, Arnold (1922), Harmonielehre, 3. Aufl., Wien: Universal Edition. (1958), Briefe, hg. von Erwin Stein, Mainz; Schott. 
Šín, Otakar (1927), „Hábova Nová nauka o harmonii« [Hábas Neue Harmonielehre], Hudební rozhledy 3/7, 125-130; 3/8, 143-146.

Skuherský, František Zdeněk (1885), Harmonielehre auf wissenschaftlicher Grundlage, Prag: Fr. Urbánek.

Weigl, Bruno (1925), Die Lehre von der Harmonik der diatonischen, der ganztonigen und der chromatischen Tonreihe, Mainz u.a.: B. Schott's Söhne. 